\title{
A randomized phase II trial of intra-arterial chemotherapy using SM-11355 (Miriplatin) for hepatocellular carcinoma
}

\author{
Takuji Okusaka • Hiroshi Kasugai • Hiroshi Ishii • \\ Masatoshi Kudo • Michio Sata - Katsuaki Tanaka • \\ Yasukazu Shioyama • Kazuaki Chayama • \\ Hiromitsu Kumada • Masaharu Yoshikawa • \\ Toshihito Seki • Hidetugu Saito • Naoaki Hayashi • \\ Keiko Shiratori - Kiwamu Okita • Isao Sakaida • \\ Masao Honda • Yukio Kusumoto • Takuya Tsutsumi • \\ Kenji Sakata
}

Received: 10 October 2011 / Accepted: 27 November 2011 / Published online: 21 December 2011

(C) The Author(s) 2011. This article is published with open access at Springerlink.com

\begin{abstract}
Background SM-11355 is a platinum complex developed to treat hepatocellular carcinoma (HCC) via administration into the hepatic artery as a sustained-release suspension in iodized oil. We conducted a multicenter phase II trial in patients with HCC to evaluate the efficacy and safety of SM-11355, using a Zinostatin stimalamer
\end{abstract}

\author{
T. Okusaka $(\bowtie)$ \\ Hepatobiliary and Pancreatic Oncology Division, National Cancer \\ Center Hospital, \\ 5-1-1 Tsukiji, \\ Chuo-ku, Tokyo 104-0045, Japan \\ e-mail: tokusaka@ncc.go.jp \\ H. Kasugai \\ Department of Gastrointestinal Oncology, Osaka Medical Center \\ for Cancer and Cardiovascular Diseases, \\ Osaka, Japan \\ e-mail: kasugai-clinic@lime.plala.or.jp \\ H. Ishii \\ Hepatobiliary and Pancreatic Section, Gastroenterological \\ Division, Cancer Institute Hospital, \\ Tokyo, Japan \\ e-mail: hiroshi.ishii@jfcr.or.jp \\ M. Kudo \\ Department of Gastroenterology and Hepatology, Kinki \\ University, \\ Osaka, Japan \\ e-mail: m-kudo@med.kindai.ac.jp \\ M. Sata \\ Division of Gastroenterology, Kurume University, \\ Fukuoka, Japan \\ e-mail: msata@med.kurume-u.ac.jp
}

suspension in iodized oil as a reference. Methods Patients with unresectable HCC were randomized 2:1 to receive administration of the SM-11355 or Zinostatin stimalamer suspension into the hepatic artery. A second injection was given 4-12 weeks later. Efficacy was evaluated by CT 3 months after treatment and categorized as therapeutic

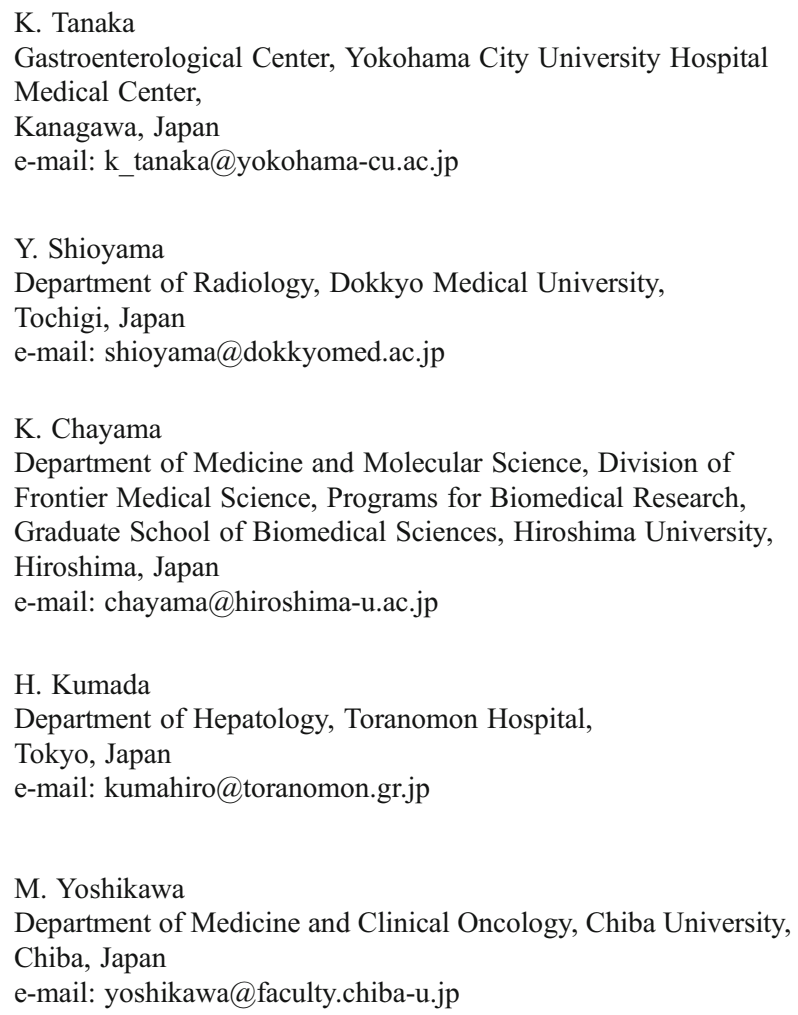


effect (TE) V to I, where TE V was defined as disappearance or $100 \%$ necrosis of all treated tumors. Results A total of 122 patients were evaluated for efficacy and toxicity (SM$11355, n=83$; Zinostatin stimalamer, $n=39$ ). Baseline characteristics were similar in the two groups. The TE V rates were $26.5 \%(22 / 83)$ and $17.9 \%$ (7/39) in the SM-11355 and Zinostatin stimalamer groups, respectively. In the SM11355 group,the most frequent drug-related adverse events (AEs) of $\geq$ grade 3 were elevated AST, elevated ALT, thrombocytopenia, and hyperbilirubinemia. The AEs with the largest difference between the two groups (SM-11355 vs. Zinostatin stimalamer) were hepatic vascular injury ( 0 vs.

T. Seki

Department of Gastroenterology and Hepatology, Kansai Medical University Takii Hospital,

Osaka, Japan

e-mail: sekit@takii.kmu.ac.jp

H. Saito

Department of Internal Medicine, School of Medicine, Keio University Hospital,

Tokyo, Japan

e-mail: hsaito@a2.keio.ac.jp

N. Hayashi $\cdot$ K. Shiratori

Department of Gastroenterology, Tokyo Women's Medical

University Hospital,

Tokyo, Japan

K. Shiratori

e-mail: tskeiko@ige.twmu.ac.jp

K. Okita $\cdot$ I. Sakaida

Department of Gastroenterology and Hepatology, Yamaguchi University Graduate School of Medicine,

Yamaguchi, Japan

K. Okita

e-mail: icb68895@nifty.com

I. Sakaida

e-mail: sakaida@yamaguchi-u.ac.jp

M. Honda

Department of Gastroenterology, Kanazawa University Graduate School of Medicine,

Kanazawa, Japan

e-mail: mhonda@m-kanazawa.jp

Y. Kusumoto $\cdot$ T. Tsutsumi

Department of Internal Medicine, Nagasaki Municipal Hospital, Nagasaki, Japan

Y. Kusumoto

e-mail: kusumoto@nmh.jp

T. Tsutsumi

e-mail: naika@nmh.jp

K. Sakata

Department of Gastroenterology, Omuta General Hospital, Omuta, Japan
$48.4 \%$ ) and eosinophilia ( 84.3 vs. $41.0 \%$ ). The 2 -year and 3year survival rates were $75.9 \%$ vs. $70.3 \%$ and $58.4 \%$ vs. $48.7 \%$, respectively. Conclusions The results suggest that SM-11355 in iodized oil has similar efficacy to Zinostatin stimalamer and that repeated dosing of SM-11355 is possible without hepatic vascular injury in cases of relapse.

Keywords Iodized oil · MIRIPLA $\cdot$ Liver cancer . Suspension · Parallel study

\section{Introduction}

International cancer statistics from 2002 indicate that hepatocellular carcinoma (HCC) ranks third behind lung and gastric cancer in the number of deaths [1]. The impact of current standard treatments for advanced HCC, including conventional transcatheter arterial chemoembolization (TACE) using doxorubicin or cisplatin is limited and the prognosis is unsatisfactory [2]. Therefore, there is a clear need for new treatments in management of this disease.

SM-11355, (SP-4-2)-[(1R,2R)-cyclohexane-1,2-diamine$\left.N, N^{\prime}\right]$ bis (tetradecanoato- $O$ ) platinum monohydrate (Fig. 1) is a highly lipophilic platinum derivative that can be delivered suspended in iodized oil, an oily lymphographic agent, via injection into the hepatic artery [3]. Following injection into an HCC-feeding artery, iodized oil selectively accumulates in the tumor. Similarly, an iodized oil suspension of SM-11355 accumulates selectively within HCC nodules, allowing continuous release of active platinum compounds into tumor tissues. A phase I dose-finding study using different injection levels indicated a recommended dose of $20 \mathrm{mg} / \mathrm{mL}$ and an upper limit of the injection volume of $6 \mathrm{~mL}$ [4]. In an early phase II trial, SM-11355 showed a promising anticancer effect with a mild toxicity profile in patients with advanced HCC. Responses were evaluated by computed tomography (CT) three months after treatment, with complete response (CR) defined as disappearance or $100 \%$ necrosis of all tumors. Iodized oil accumulation in tumors was taken to indicate necrosis. Of 16 eligible patients, 9 (56\%) showed CR [5]. This CR rate was superior to our expectation, because the $\mathrm{CR}$ rate in conventional TACE is $15-20 \%$ based on the same evaluation criteria [6, 7]. Therefore, the results of the early phase II study

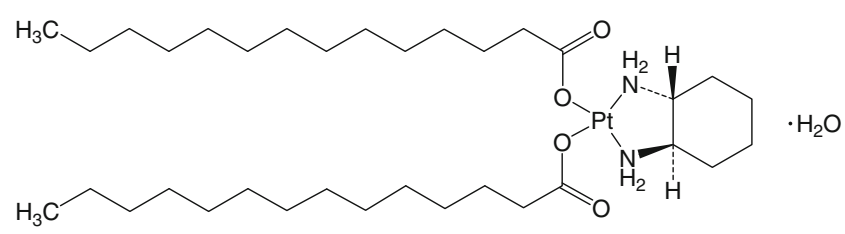

Fig. 1 Structural formula of SM-11355 
indicated that SM-11355 has potential as an alternative to TACE in treatment of advanced HCC.

Based on these findings, we conducted a late phase II open-label trial of SM-11355. The aims of the study were to re-evaluate the efficacy, safety and pharmacokinetics of SM11355 in a larger population, since only 16 eligible patients were included in the previous phase II study, and to confirm the candidacy of SM-11355 as an experimental treatment in a forthcoming clinical study in comparison with conventional TACE. To achieve regulatory approval of SM-11355 in Japan, it was necessary to undertake a parallel study. Therefore, we conducted a randomized phase II trial using Zinostatin stimalamer as a reference, because this agent is the only commercially available lipophilic drug for HCC in Japan and chemolipiodolization of Zinostatin stimalamer has been approved for treatment of advanced HCC in Japan $[8,9]$. However, statistical comparisons between the two treatment groups were not planned since the goal of the study was re-evaluation of outcomes for SM-11355, and because the sample size required to conduct a statistical analysis was larger than expected.

\section{Patients and methods}

Inclusion criteria

Consecutive patients with HCC were eligible for the study if they had no indication for resection or local ablation therapy. The diagnosis was confirmed histologically and/or clinically using angiography and enhanced CT. Each patient was required to meet the following criteria: at least one measurable intrahepatic lesion that showed tumor staining by CT; tumor stage II or III in the staging system of the Liver Cancer Study Group of Japan [6, 7]; Child-Pugh classification $\mathrm{A}$ or $\mathrm{B}$; adequate hematological function (WBC $\geq 3000 / \mu \mathrm{L}$, blood platelets $\geq 50000 / \mu \mathrm{L}$, hemoglobin $\geq 9.5 \mathrm{~g} / \mathrm{dL}$ ), adequate hepatic function (AST and ALT $\leq$ 5 -fold the upper limit of normal, serum bilirubin $<3 \mathrm{mg} / \mathrm{dL}$, serum albumin $\geq 3 \mathrm{~g} / \mathrm{dL}$ ), adequate renal function (serum creatinine $\leq$ the upper limit of normal); an Eastern Cooperative Oncology Group performance status of 0-2; age 20 to 74 years old; minimum life expectancy $\geq 3$ months, and provision of written informed consent. Patients who had undergone hepatic resection, local ablation therapy, and/or TACE were eligible if they showed no evidence of local tumor recurrence in the treated lesions. Patients who had undergone chemolipiodolization with anti-cancer agents other than Zinostatin stimalamer or a platinum-containing agent were also eligible if the treated lesions were resected. The previous anticancer treatment had to have been discontinued for at least 4 weeks before enrollment in this study.

\section{Exclusion criteria}

Patients were excluded if they met any of the following criteria: history of allergy to iodine-containing agents and/ or contrast material; history of systemic chemotherapy; serious complication such as a cardiac disease or a thyroid disease ; concomitant malignancy; bile duct invasion; pregnant or lactating women and fertile patients who were not using effective contraception; and participation in another trial within 6 months before giving informed consent.

\section{Study treatment}

Patients who met the entry criteria were provisionally registered and randomly assigned to the SM-11355 or Zinostatin stimalamer group before undergoing angiography. Each investigator then confirmed registration after establishing that the patient met the following additional requirements based on angiographic findings: intrahepatic lesions that showed tumor staining and were fed by an artery with an appropriate structure for catheter insertion; no evidence of tumor thrombus in the portal or hepatic vein; no evidence of intrahepatic arteriovenous shunting; and no evidence of local tumor recurrence in previously treated lesions. The central random assignment by dynamic allocation to either a SM-11355 group or Zinostatin stimalamer group was stratified according to center and maximun tumor diameter.

A suspension of SM-11355 (MIRIPLA; Dainippon Sumitomo Pharma Co., Japan) or Zinostatin stimalamer (SMANCS; Astellas Pharma Inc., Japan) in iodized oil was injected into the hepatic artery using Seldinger's technique. Patients in the SM-11355 group received SM-11355 suspended in iodized oil $(20 \mathrm{mg} / \mathrm{mL})$ in a volume of up to $6 \mathrm{~mL}$ according to tumor size. Patients in the Zinostatin stimalamer group received Zinostatin stimalamer suspended in iodized oil ( $1 \mathrm{mg}$ titer $/ \mathrm{mL}$ ) in a volume of up to $6 \mathrm{~mL}$. When iodized oil accumulation in the treated tumor was insufficient and tumor staining was found in diagnostic imaging 5 weeks ( \pm 10 days) after the first injection, a second injection was given within 12 weeks after the first injection.

\section{Efficacy and safety assessment}

The antitumor effect was evaluated by CT or MRI 3 months after the last injection according to the response criteria proposed by the Liver Cancer Study Group of Japan [10], which are similar to the criteria proposed by the European Association for the Study of the Liver (EASL) Panel of Experts on HCC [11]. Tumor size was measured using the sum of the products of the perpendicular longest diameters of all measurable lesions. In the response evaluation criteria, iodized oil accumulation in a tumor is regarded as an indication of necrosis because significant positive correlations 
have been reported between iodized oil accumulation observed on CT images and necrotic regions in resected tumors examined pathologically after TACE and after intra-arterial chemotherapy with iodized oil $[5,8,12,13]$. Therapeutic effect (TE) was defined as follows: TE V, disappearance or $100 \%$ necrosis of all treated tumors; TE $\mathrm{IV}$, more than $50 \%$ reduction in tumor size and/or more than $50 \%$ necrosis; TE III, more than $25 \%$ reduction in tumor size and/or more than $25 \%$ necrosis; and TE I, more than $25 \%$ increase in tumor size regardless of the necrotic effect. TE II was defined as a response not qualifying for classification as TE V, IV, III, or I. When a patient assigned to the SM-11355 group and judged to be TE V developed a tumor in a different region and requested SM-11355, the drug was given continuously after the study, provided that this was felt to be necessary by the investigator. The primary endpoint was the TE V rate. The secondary endpoints were the response rate based on the Response Evaluation Criteria in Solid Tumors (RECIST) and on the Japan Society for Cancer Therapy Criteria [14], which are similar to the World Health Organization (WHO) Criteria. The serum $\alpha$ fetoprotein (AFP) level of each patient was measured before and 5 weeks after each treatment. Survival was evaluated using the Kaplan-Meier method. Toxicity was assessed according to the criteria of the Japan Society for Cancer Therapy [15], which are also fundamentally similar to WHO criteria.

\section{Pharmacokinetics}

Pharmacokinetic data were determined in patients in the SM-11355 group who gave written informed consent and were treated at institutions where a pharmacokinetic study could be conducted. Peripheral blood samples $(5 \mathrm{ml})$ were collected 3 weeks after each treatment for determination of the total plasma platinum concentration and the platinum concentration in methanol extracts (SM-11355 metabolite concentration). The total platinum concentration in resected tissue was also determined in a patient who underwent surgery after evaluation of efficacy.

\section{Statistical analysis}

We anticipated enrollment of 120 patients at 17 participating hospitals over the study period of 3 years. A 2:1 ratio for SM-11355 to Zinostatin stimalamer randomization was chosen as a balance between the goals of the study, which were to re-evaluate the efficacy, safety and pharmacokinetics of SM-11355 in a larger population than that in the previous phase II study, and the current limited use of Zinostatin stimalamer. The number of subjects was determined based on the feasibility of the study because the sample size required to conduct a statistical analysis was larger than expected. Assuming a baseline $15 \% \mathrm{TE} \mathrm{V}$ rate for conventional TACE [6], the SM-11355 arm would be considered 'favorable' if there was a $10 \%$ improvement in this endpoint (to $25 \%$ ) with an acceptable toxicity profile. A total of 80 patients in the SM-11355 arm is needed to estimate the TE-V rate with an accuracy of $\pm 10 \%$.

This study was not powered to permit formal statistical comparison between the two treatment arms. However, it does allow an initial assessment of SM-11355 in terms of $\mathrm{TE}-\mathrm{V}$, response rate, overall survival and toxicity with a view to performance of a follow-on phase III study.

\section{Results}

Patient

From April 2002 to October 2004, 131 patients were enrolled in the study, and 126 were assigned randomly at a 2:1 ratio to receive SM-11355 (85 patients) or Zinostatin stimalamer (41 patients) (Fig. 2). Five patients were excluded from the randomization because tumor staining was not observed in angiography and/or an appropriate hepatic artery for selective catheter insertion was not found $(n=3)$, multiple tumors were observed in angiography that required reconsideration of the treatment strategy $(n=1)$, and withdrawal of consent $(n=1)$. After administration, 4 patients were identified as ineligible due to a platelet count $<50,000 / \mu \mathrm{L}(n=1)$, esophageal cancer $(n=1)$ in the SM-11355 group, and deviation from correct use of the investigational products $(n=2)$ in the Zinostatin stimalamer group. Therefore, 122 patients (SM-11355 group, $n=$ 83; Zinostatin stimalamer group, $n=39$ ) were analyzed for efficacy and safety. The baseline demographic and disease characteristics of the patients are listed in Table 1.

Of the 85 original patients in the SM-11355 group, 18 were withdrawn from the study before the planned evaluation of efficacy 3 months after the first injection because of marked progression of the primary disease $(n=5)$, serious adverse events $(n=4)$, use of prohibited concomitant therapeutic agents or a requirement for combination therapy $(n=$ 3 ), and other reasons (duplicated count). Treatment was terminated in 11 patients after evaluation of the the first injection because complete necrosis of tumors (TE V) was obtained. The remaining 56 patients received a second injection.

Of the 41 patients in the Zinostatin stimalamer group, 9 were withdrawn before the planned evaluation of efficacy 3 months after the first injection, due to marked progression of the primary disease $(n=2)$, serious adverse events $(n=1)$, contravention of the protocol $(n=1)$, appearance of hepatic injury $(n=1)$, and other reasons (duplicated count). Treatment was terminated in 7 patients after evaluation of the first injection because complete necrosis of tumors (TE V) was obtained. The remaining 25 patients received a second injection. 


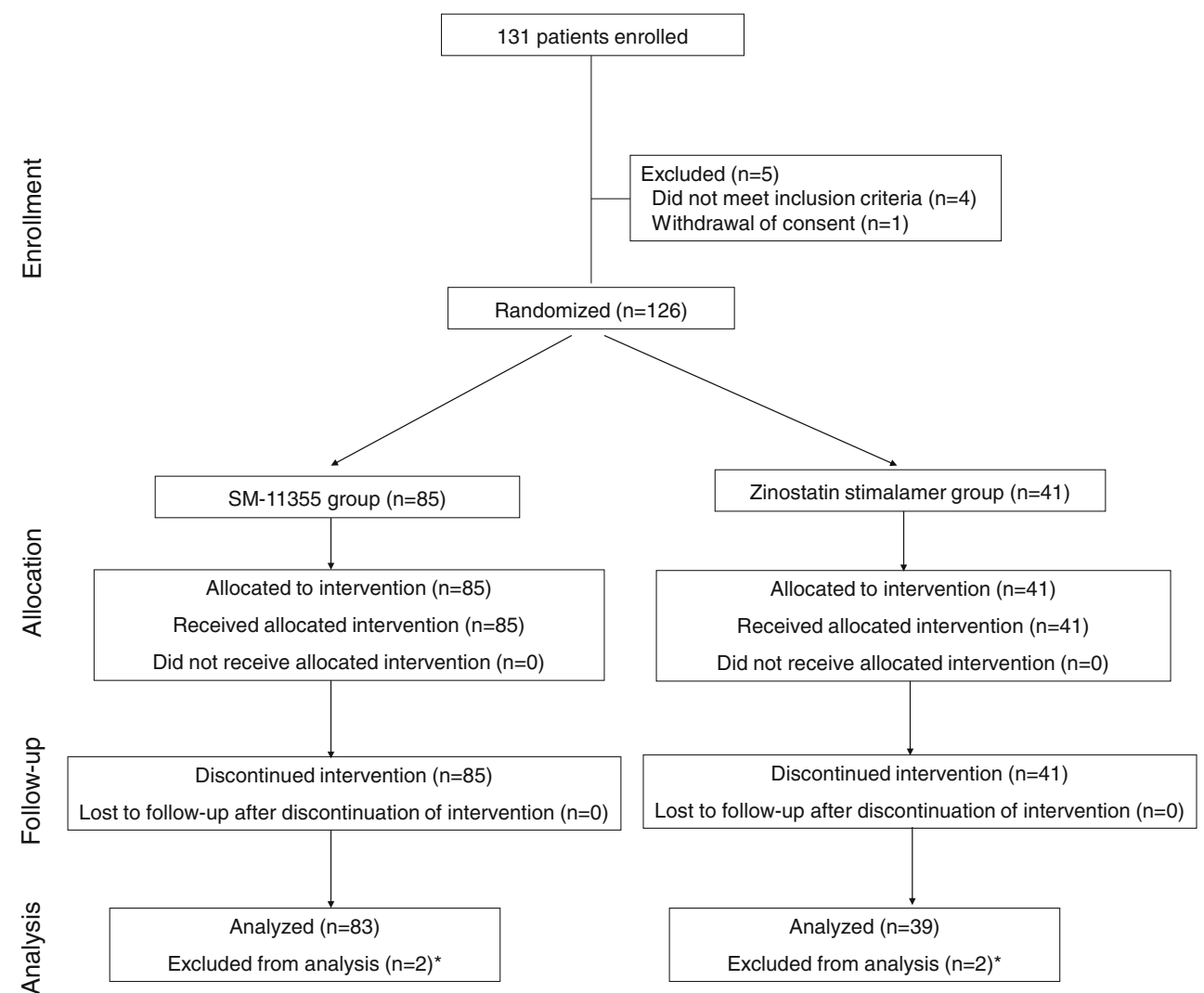

The second injection was given to 56 patients in the SM-11355 group and to $\mathbf{2 5}$ patient in the Zinostatin stimalamer group

*Two of the patients each in the both groups were excluded from the full analysis set defined in the protocol. Refer to patient characteristics in results.

Fig. 2 Study flow diagram

Table 1 Patient background

\begin{tabular}{lll}
\hline & SM-11355 & Zinostatin stimalamer \\
\hline Number of patients & 83 & 39 \\
Sex (male:female) & $70: 13(84.3 \%: 15.7 \%)$ & $30: 9(76.9 \%: 23.1 \%)$ \\
Age (median) & $67.0(48-74)$ & $68.0(52-74)$ \\
PS (0:1:2:3:4) & $80: 3: 0: 0: 0$ & $35: 4: 0: 0: 0$ \\
HBs antigen positive & $9(13.6 \%)$ & $1(3.2 \%)$ \\
HCV antibody positive & $55(83.3 \%)$ & $30(96.8 \%)$ \\
HBs antigen · HCV antibody positive & $2(3.0 \%)$ & $0(0 \%)$ \\
Tumor stage (I:II:III:IV-A:IV-B) & $0: 43: 40: 0: 0$ & $0: 19: 20: 0: 0$ \\
Child-Pugh Classification (A:B:C) & $61: 22: 0$ & $32: 7: 0$ \\
Previously treated & $25(30.1 \%)$ & $13(33.3 \%)$ \\
Number of tumors 1 & $24(28.9 \%)$ & $9(23.1 \%)$ \\
2 & $19(22.9 \%)$ & $11(28.2 \%)$ \\
3 & $16(19.3 \%)$ & $7(17.9 \%)$ \\
$\geq 4$ & $24(28.9 \%)$ & $12(30.8 \%)$ \\
Maximum tumor diameter (mm) (Min-Max) & $29.0(10.0-80.0)$ & $29.0(10.0-94.0)$ \\
\hline
\end{tabular}


Table 2 Antitumor efficacy

\begin{tabular}{|c|c|c|c|c|c|c|c|c|}
\hline Group & $\mathrm{N}$ & \multicolumn{7}{|c|}{ Antitumor efficacy } \\
\hline \multicolumn{9}{|c|}{ “Criteria for Evaluation of Direct Effects on Hepatocellular Carcinoma” of the Liver Cancer Study Group of Japan } \\
\hline & & $\mathrm{V}$ & IV & III & II & I & $\mathrm{NE}$ & Percentage of TE V (\%) $[95 \% \mathrm{CI}]$ \\
\hline SM-11355 & 83 & 22 & 21 & 12 & 7 & 17 & 4 & $26.5[17.4-37.3]$ \\
\hline Zinostatin stimalamer & 39 & 7 & 14 & 4 & 10 & 1 & 3 & $17.9[7.5-33.5]$ \\
\hline \multicolumn{9}{|c|}{ Response Evaluation Criteria in Solid Tumors (RECIST) } \\
\hline & & $\mathrm{CR}$ & PR & SD & & PD & $\mathrm{NE}$ & Percentage of $\mathrm{CR}+\mathrm{PR}$ \\
\hline SM-11355 & 83 & 0 & 20 & 52 & & 10 & 1 & $24.1[15.4-34.7]$ \\
\hline Zinostatin stimalamer & 39 & 0 & 10 & 23 & & 6 & 0 & $25.6[13.0-42.1]$ \\
\hline \multicolumn{9}{|c|}{ “Clinical Response Evaluation Criteria for Solid Tumor Chemotherapy” of the Japan Society for Cancer Therapy } \\
\hline & & CR & PR & MR & $\mathrm{NC}$ & PD & $\mathrm{NE}$ & Percentage of $\mathrm{CR}+\mathrm{PR}$ \\
\hline SM-11355 & 83 & 0 & 17 & 10 & 36 & 19 & 1 & $20.5[12.4-30.8]$ \\
\hline Zinostatin stimalamer & 39 & 0 & 9 & 5 & 19 & 6 & 0 & $23.1[11.1-39.3]$ \\
\hline
\end{tabular}

\section{Efficacy}

The antitumor efficacy is shown in Table 2. The percentages of TE V patients were 26.5\% (22/83) [95\% confidence interval (CI): $17.4-37.3 \%$ ] in the SM-11355 group and $17.9 \%(7 / 39)$ [95\% CI: 7.5-33.5\%] in the Zinostatin stimalamer group. In a RECIST assessment, response rates were 24.1\% (20/83) [95\% CI: 15.4-34.7\%] and 25.6\% (10/39) [95\% CI: $13.0-42.1 \%$ ] in the respective groups. Based on the Japan Society for Cancer Therapy Criteria, the tumor responses were 20.5\% (17/83) [95\% CI: $12.4-30.8 \%$ ] and $23.1 \%$ (9/39) [95\% CI: 11.1-39.3\%] in the respective groups (Table 2).

Of 61 patients with a pre-treatment AFP level above the upper limit of normal in the SM-11355 group, 6 / 60 (10\%) had an AFP level within the normal range 5 weeks after the first injection. No data for the AFP level were available for 1 patient in the SM-11355 group at 5 weeks after the first injection. Among the 61 patients, 37 received a second injection and $6(16 \%)$ had a normal AFP level 5 weeks after the second injection. Of the 26 patients in the Zinostatin stimalamer group with a pre-treatment AFP level above the upper limit of normal, none had an AFP level within the normal range 5 weeks after the first injection. Among the 26 patients, 18 received a second injection, but none had a normal AFP level 5 weeks after the second injection.

Cumulative survival rates are shown in Fig. 3. The follow-up period was approximately 3 years after the treatment period. The longest follow-up periods in the SM11355 and Zinostatin stimalamer groups were both 5.6 years, and the median periods were 3.0 years and 2.8 years, respectively. The one-year survival rates in the SM-11355 and

Fig. 3 Cumulative survival rate

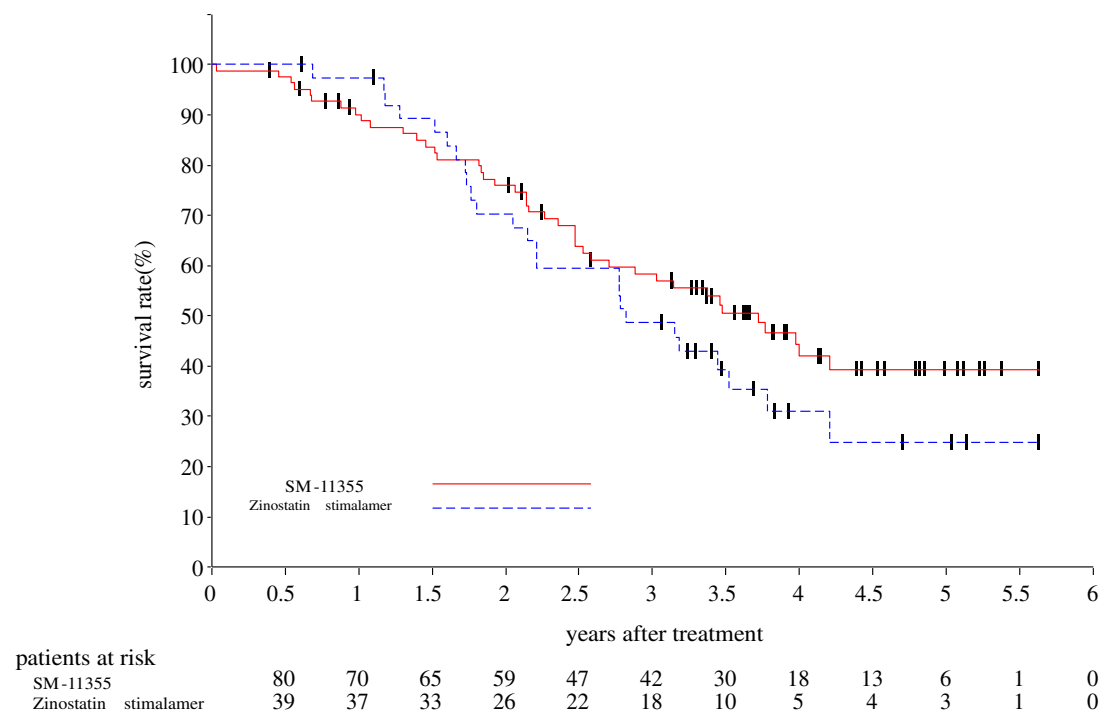


Table 3 Hematological and non-hematological adverse events

\begin{tabular}{|c|c|c|c|c|c|c|}
\hline & \multicolumn{3}{|l|}{ SM-11355 } & \multicolumn{3}{|c|}{ Zinostatin stimalamer } \\
\hline & No. of patients & All (\%) & $\geq$ Grade $3(\%)$ & No. of patients & All (\%) & $\geq$ Grade $3(\%)$ \\
\hline Decrease in leukocytes & 83 & 41.0 & 1.2 & 39 & 66.7 & 0 \\
\hline Decrease in lymphocytes & 83 & 79.5 & 0 & 39 & 79.5 & 0 \\
\hline Decrease in neutrophils & 83 & 53.0 & 8.4 & 39 & 43.6 & 2.6 \\
\hline Decrease in platelets & 83 & 50.6 & 12.0 & 39 & 74.4 & 10.3 \\
\hline Decrease in hemoglobin & 83 & 15.7 & 0 & 39 & 10.3 & 0 \\
\hline Increase in eosinophils & 83 & 84.3 & 0 & 39 & 41.0 & 0 \\
\hline Increase in monocytes & 83 & 57.8 & 0 & 39 & 76.9 & 0 \\
\hline Fatigue & 83 & 39.8 & 0 & 39 & 46.2 & 0 \\
\hline Fever & 83 & 96.4 & 3.6 & 39 & 97.4 & 0 \\
\hline Chills & 83 & 39.8 & 0 & 39 & 51.3 & 0 \\
\hline Vomiting & 83 & 55.4 & 1.2 & 39 & 51.3 & 0 \\
\hline Pain at injection site & 83 & 43.4 & 0 & 39 & 41.0 & 2.6 \\
\hline Decrease in albumin & 83 & 50.6 & 0 & 39 & 28.2 & 0 \\
\hline Increase in ALP & 83 & 30.1 & 1.2 & 39 & 51.3 & 0 \\
\hline Increase in ALT & 83 & 59.0 & 24.1 & 39 & 66.7 & 20.5 \\
\hline Increase in AST & 83 & 62.7 & 26.5 & 39 & 79.5 & 38.5 \\
\hline Increase in bilirubin & 83 & 57.8 & 12.0 & 39 & 71.8 & 5.1 \\
\hline Decrease in calcium & 83 & 38.6 & 0 & 39 & 51.3 & 0 \\
\hline Increase in $\gamma$-GTP & 83 & 49.4 & 0 & 39 & 61.5 & 0 \\
\hline Increase in glycemia & 83 & 56.6 & 12.0 & 39 & 56.4 & 5.1 \\
\hline Increase in LDH & 83 & 60.2 & 0 & 39 & 69.2 & 0 \\
\hline Increase in CRP & 83 & 95.2 & 0 & 39 & 79.5 & 0 \\
\hline Prolonged PT time & 83 & 42.2 & 1.2 & 39 & 28.2 & 0 \\
\hline Decrease in urinary creatinine & 83 & 54.2 & 0 & 39 & 56.4 & 0 \\
\hline Increase in urinary creatinine & 83 & 49.4 & 0 & 39 & 38.5 & 0 \\
\hline Increase in urinary NAG & 83 & 89.2 & 0 & 39 & 87.2 & 0 \\
\hline
\end{tabular}

Adverse events that occurred at a rate of $>40 \%$ are shown

Zinostatin stimalamer groups were $90.1 \%$ and $97.4 \%$, the 2year survival rates were $75.9 \%$ and $70.3 \%$, respectively, and the 3-year survival rates were $58.4 \%$ and $48.7 \%$, respectively. The median survival time (MST) was 3.7 years in the SM-11355 group and 2.8 years in the Zinostatin stimalamer group.

\section{Safety}

Hematological adverse events were relatively mild and transient in both groups (Table 3). The incidences of neutropenia and decreased hemoglobin were similar in the two groups, but the incidence of eosinophilia was higher in the SM-11355 group, and the incidences of leukopenia and thrombocytopenia were higher in the Zinostatin stimalamer group. Most non-hematological adverse events (Table 3) were also mild and transient in both groups. Major events of grade 3 or higher involved liver dysfunction (including elevations in AST, ALT and hyperbilirubinemia) and hyperglycemia, but these had similar incidences in both groups and most were reversible.

One patient in the SM-11355 group died of esophageal variceal rupture, which occurred 12 days after the first injection, and one patient in the Zinostatin stimalamer group died of hepatic failure 168 days after the second injection. Esophageal variceal rupture was considered not to be related to the treatment because the condition was recognized before initiation of treatment and the event was not classified as a toxicity. Other serious adverse events occurred in 8 patients in the SM11355 group (increase in AST in 2 patients; and increase in ALT, sepsis, systemic inflammatory response syndrome (SIRS: a syndrome characterized by systemic inflammation and extensive tissue damage associated with serious infection), decrease in neutrophils, acute myocardial infarction (AMI), and hypotension in 1 case each) and in 2 patients in the Zinostatin stimalamer group (respiratory distress and arrhythmia, and abdominal pain in 1 case each). All the patients recovered with appropriate treatment. Most of these events 
were considered to be probable or possible drug-related toxicities, except for the cases of SIRS and AMI in the SM-11355 group. SIRS was judged to have no association with the investigational drug based on the results of blood culture and changes in test values. This patient was treated using a urinary catheter, and urinary tract infection is a cause of SIRS. A similar judgment was made for the case of AMI based on the chronological relationship between drug administration and the onset of disease.

In the subsequent angiographic examination before the second administration of SM-11355 or Zinostatin stimalamer or in postprotocol treatment, hepatic artery damage that was probably due to intra-arterial drug administration was observed in $15 / 31$ (48.4\%) patients, shunt occurred in 5/ $31(16.1 \%)$, and disorders of the hepatobiliary system were observed in 3/39 (7.7\%) in the Zinostatin stimalamer group. None of these events were observed in patients in the SM11355 group. Grade 3 hepatic artery damage and a grade 4 disorder of the hepatobiliary system were observed in 1 case each in the Zinostatin stimalamer group. Hepatobiliary damage that may have been caused by arterial damage was found in 3 patients in the Zinostatin stimalamer group (1 case each of liver atrophy and bile duct dilatation, bile duct necrosis, and liver failure and bile duct stricture), whereas there were no such findings in the SM-11355 group.

In the SM-11355 group, the percentages of patients with an increase in Child-Pugh score of one or more points compared to the pre-administration score were $27.7 \%$ (23/83) and $17.9 \%$ $(10 / 56)$ in the 5 weeks after the 1 st administration and the 5 weeks after the 2 nd administration, respectively. In the Zinostatin stimalamer group, these percentages were 35.9\% $(14 / 39)$ and $50.0 \%$ (12/24), respectively (Fig. 4).

\section{Pharmacokinetics}

Total plasma platinum concentrations and platinum concentrations in methanol extracts (Table 4) were determined in 30 and 24 patients in the SM-11355 group who were given one

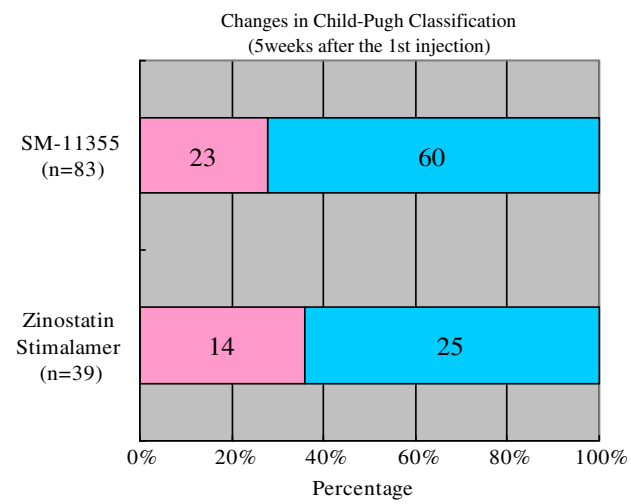

$\square$ Deteriorated $\square$ Stable or improved and two injections, respectively, and received median doses of 85 (Min-max: 24-120) and $120(10-120) \mathrm{mg}$, respectively. The mean total platinum concentrations after the first and second injections were 9.6 and $12.9 \mathrm{ng} / \mathrm{mL}$, respectively, and the mean percentages of the concentration in methanol extracts relative to the total plasma platinum concentration were $12.2 \%$ and $9.8 \%$ after the first and second injections, respectively. In one patient who underwent surgery 172 days after the second injection, the total platinum concentration was determined in the resected liver tissue. The total dose was $200 \mathrm{mg}$ (first injection: $100 \mathrm{mg}$; second injection: $100 \mathrm{mg}$ ) and the concentration in the tumor region of sample S6, which had a $10 \%$ necrotic effect, was $62,000 \mathrm{ng} / \mathrm{g}$ tissue and that in the non-tumor region was $22,000 \mathrm{ng} / \mathrm{g}$ tissue. In contrast, the concentration in the tumor region of sample S8, which showed $50 \%$ necrosis, was $260,000 \mathrm{ng} / \mathrm{g}$ tissue and that in the non-tumor region was $67,000 \mathrm{ng} / \mathrm{g}$ tissue.

\section{Discussion}

Most anticancer agents used in TACE are water-soluble and inappropriate for suspension in iodized oil, and are usually administered as a water-in-oil emulsion. Consequently, these agents have reduced sustained release due to poorer retention in the tumor, leading to a limited antitumor effect and adverse effects caused by diffusion of the agents into the blood [16]. In contrast, lipophilic anticancer agents have a high affinity for iodized oil and those injected into the hepatic artery with iodized oil are retained selectively in tumors and exert continuous antitumor effects. SM-11355 is a structurally modified platinum complex with improved affinity for iodized oil due to increased lipophilicity [3]. In an AH109A-transplanted rat liver tumor model, the platinum concentration in the tumor was sustained for longer following administration of a iodized oil suspension of SM-11355 compared to a suspension of cisplatin, with SM-11355 distributed in tumor tissues more selectively than cisplatin [17]. Phase I and early phase II trials

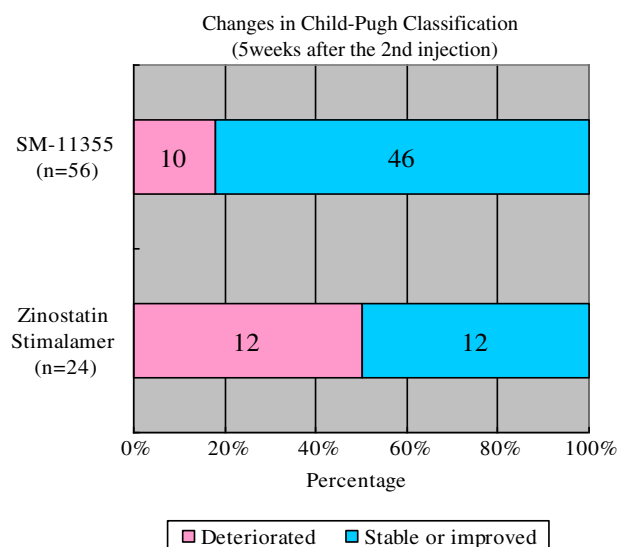

Fig. 4 Changes in Child-Pugh Classification 
Table 4 Blood drug concentrations

\begin{tabular}{llll}
\hline Administration frequency & & Once & Twice \\
\hline Dose (mg) & Number of patients & $30^{*}$ & $24 *$ \\
& Median (Min-Max) & $85.0(24-120)$ & $120.0(10-120)$ \\
Total plasma platinum concentration $(\mathrm{ng} / \mathrm{mL})$ & Number of patients & 30 & 24 \\
& Mean & 9.6 & 32.9 \\
SM-11355 metabolite concentration in methanol extracts $(\mathrm{ng} / \mathrm{mL})$ & Number of patients & 32 & 1.17 \\
[SM-11355 in methanol-extracted fraction*]/ & Mean & 30 & 24 \\
[total plasma platinum concentration] $\times 100(\%)$ & Number of patients & 12.2 & 9.8 \\
\hline
\end{tabular}

Number of subjects in whom both the total plasma platinum concentration and SM-11355 metabolite concentration in methanol extracts were measured

* Methanol-extracted fraction: The fraction of SM-11355-derived substances includes components that may exert therapeutic activity as an anticancer agent and excludes components that are irreversibly bound to plasma protein

of SM-11355 have also shown that the total plasma platinum concentration is much lower than that with cisplatin $[4,5,18]$. Our pharmacokinetic data verify these results and suggest that SM-11355 is retained in liver tumors selectively and exerts a continuous effect on the tumor.

In patients in whom the total plasma platinum concentration and the platinum concentration in methanol extracts were determined after the first and second injections, the platinum concentration in methanol extracts 3 weeks after injection (estimated to be the peak of the total plasma platinum concentration) was approximately $10 \%$ of the total plasma platinum concentration. Of the platinum components released from the SM-11355 suspension and transferred into the systemic circulation, some are irreversibly bound to plasma proteins and are no longer bioactive. After exclusion of these components, the amount remaining in the plasma is estimated to be up to about $10 \%$ of the dose. The total platinum concentrations in several regions of the liver were also determined in one patient. The concentrations in tumors regions were significantly higher than those in non-tumor regions and several thousand-fold higher than the mean total plasma platinum concentration at 3 weeks \pm 3 days after the second injection $(12.9 \mathrm{ng} / \mathrm{mL})$. The total platinum concentration was also higher in tissues in which a higher antitumor effect was observed.

The results of the efficacy re-evaluation suggested that SM11355 has a similar effect to that of Zinostatin stimalamer following injection of an iodized oil suspension of each drug into the hepatic artery. The primary endpoint (TE V rate based on the Criteria for Evaluation of Direct Effects on Hepatocellular Carcinoma) and the secondary endpoint (response rate based on the Japan Society for Cancer Therapy Criteria and RECIST) in the SM-11355 group were almost the same as those in the Zinostatin stimalamer group. However, the percentage of TE V cases in the SM-11355 group (26.5\% [17.4$37.3 \%])$ in this trial was lower than the value of $56 \%$ [30$80 \%$ ] found in the early phase II trial. The discrepancy in the percentage of TE V cases may be due to differences in the tumor burden in the two trials. Eleven (68.8\%) of 16 patients in the early phase II study had 3 or less tumors and a longest tumor diameter of $3 \mathrm{~cm}$ or less, whereas only $38(45.8 \%)$ of 83 patients in the late phase II study had these characteristics.

The major toxicities of grade 3 or higher involved liver dysfunction, including increases in AST, ALT and bilirubin, and a decrease in platelets in both groups. The incidences were similar in each group and most of the effects were reversible. An increase in eosinophils was found in $84.3 \%$ of patients in the SM-11355 group, and was considered to be a SM-11355-specific adverse event. The precise mechanism is unknown, but the finding was not thought to indicate anaphylaxis because the increase in eosinophils showed no marked correlation with an increase in $\operatorname{IgE}$ and/or allegic symptoms like wheezing. Renal disorder was transient in patients of the SM-11355 group, except for a patient with sepsis. The incidences and severity of increased blood creatinine and positive urine protein in the SM-11355 group were higher than the respective levels in the Zinostatin stimalamer group $(9 / 83,10.8 \%$ vs. $2 / 39,5.1 \%$; and $22 / 83$, $26.5 \%$ vs. $2 / 39,5.1 \%$, respectively). Based on these data, we consider that the patients were thoroughly followed up.

Injection of SM-11355 did not lead to local vascular damage and had fewer irreversible effects on the hepatobiliary system compared with Zinostatin stimalamer. Zinostatin stimalamer has been reported to have major safety problems, including hepatic arterial damage and effects on the hepatobiliary system that are irreversible and prevent repeated treatment $[5,19,20]$ Therefore, SM-11355 may be advantageous for frequent repeated treatment and maintenance of liver function. The changes in Child-Pugh Class indicated a low incidence of treatment-induced hepatic dysfunction in the SM-11355 group.

Based on the results of this trial, we conclude that SM11355 in iodized oil has similar efficacy to that of Zinostatin stimalamer, which is the only drug currently approved for chemolipiodolization for HCC in Japan. The TE V rate of $26.5 \%$ in the SM-11355 group was considered 'favorable' 
based on our assumption of a TE V rate of $15 \%$ for conventional TACE before the initiation of this study, and was equivalent or superior to the rate of about $20 \%$ found in patients receiving current standard TACE treatment in a recent report [7]. Our results also suggest that repeated dosing of SM-11355 in iodized oil is possible without development of hepatic vascular injury in a case of relapse. We are currently conducting a phase III study of intra-arterial treatment with SM-11355 in comparison with conventional TACE with epirubicin, which is designed to detect the superiority of intra-arterial treatment with SM-11355 in overall survival of TACE-naïve patients with advanced HCC (Appendix).

Acknowledgements We thank the all patients participated in this study, their families, the investigators and the study site personnel. This article is dedicated to the memory of the late Dr. Hiromasa Ishii, a principal investigator, and the late Prof. K. Kobayashi and Dr. S. Okada, who served as coordinating investigators.

Disclosures The authors report potential conflicts of interest as follow: T. Okusaka and M. Sata receive grants and research supports from Dainippon-Sumitomo, M. Kumada receives directorship compensation and travel grant from Dainippon-Sumitomo, K. Shiratori receives directorship compensation from Dainippon-Sumitomo, T. Seki has stock ownership for Dainippon-Sumitomo, H. Ishii, M. Kudo, and K. Tanaka receives other interests from Dainippon-Sumitomo. Y. Shioyama receives grants and research supports and directorship compensation from Dainippon-Sumitomo. H. Kasugai, Y. Shioyama, K. Chayama, M. Yoshikawa, H. Saito, N. Hayashi, K. Okita, I. Sakaida, M. Honda, Y. Kusumoto, K. Sakata and T. Tsutsumi report no conflicts of interest.

Funding This study was supported by Dainippon-Sumitomo Pharmaceutical Co. Ltd.

Results were presented in part at the 45th American Society of Clinical Oncology Annual Meeting, May 2009, Orlando, FL (USA).

Open Access This article is distributed under the terms of the Creative Commons Attribution Noncommercial License which permits any noncommercial use, distribution, and reproduction in any medium, provided the original author(s) and source are credited.

\section{Appendix}

Coordinating investigators

Members of the Committee for Efficacy and Safety

Members of the Committee for Efficacy Evaluation

Statistics Advisor

Investigators and Institutions

Coordinating investigators

\begin{tabular}{ll}
\hline Yasuhiko Kubo & Omuta City General Hospital \\
Kenichi Kobayashi & Kanazawa University Hospital \\
Shuichi Okada & National Cancer Center Hospital \\
\hline
\end{tabular}

Members of the Committee for Efficacy and Safety

Masaru Itakura Surugadai Clinic, Medical Corporation Shun-ai-kai

Mariko Itsubo Jikei University Hospital

Junji Shibata Shibata Internal and Gastrointestinal Clinic

Shoji Fukushima Faculty of Pharmaceutical Sciences, Kobe Gakuin University

Members of the Committee for Efficacy Evaluation

\begin{tabular}{ll}
\hline Shigetoshi Fujiyama & NTT West Kyushu Hospital \\
Yutaka Horie & Saiseikai Gotsu General Hospital \\
Fuminori Moriyasu & Tokyo Medical University Hospital \\
Hiroki Inoue & Foundation Jiaikai Imamura Hospital
\end{tabular}

Statistics Advisor

Tosiya Sato

Kyoto University School of Public Health

Investigators and institutions

\begin{tabular}{ll}
\hline Hiromasa Ishii & Keio University Hospital \\
Hidetugu Saito & Keio University Hospital \\
Shuichi Okada & National Cancer Center Hospital \\
Takuji Okusaka & National Cancer Center Hospital \\
Hiromitsu Kumada & Toranomon Hospital \\
Naoaki Hayashi & Tokyo Women's Medical University Hospital \\
Keiko Shiratori & Tokyo Women's Medical University Hospital \\
Masaharu Yoshikawa & Chiba University Hospital \\
Hiroshi Ishii & National Cancer Center Hospital East \\
Yasukazu Shioyama & Ibaraki Prefectural Central Hospital \\
Katsuaki Tanaka & Yokohama City University Hospital \\
& Medical Center \\
Masao Honda & Kanazawa University Hospital \\
Hiroshi Kasugai & Osaka Medical Center for Cancer \\
& and Cardiovascular Diseases \\
Masatoshi Kudo & Kinki University Hospital \\
Toshihito Seki & Kansai Medical University Takii Hospital \\
Kazuaki Chayama & Hiroshima University Hospital \\
Kiwamu Okita & Yamaguchi University Hospital \\
Isao Sakaida & Yamaguchi University Hospital \\
Yukio Kusumoto & Nagasaki Municipal Hospital \\
Takuya Tsutsumi & Nagasaki Municipal Hospital \\
Michio Sata & Kurume University Hospital \\
Kenji Sakata & Omuta City General Hospital \\
\hline
\end{tabular}

\section{References}

1. Parkin DM, Bray F, Ferlay J, Pisani P (2005) Global cancer statistics, 2002. CA Cancer J Clin 55(2):74-108 
2. Lopez PM, Villanueva A, Llovet JM (2006) Systematic review: evidence-based management of hepatocellular carcinoma: an updated analysis of randomized controlled trials. Aliment Pharmacol Ther 23(11):1535-1547

3. Maeda M, Uchida NA, Sasaki T (1986) Liposoluble platinum(II) complexes with antitumore activity. Jpn J Cancer Res 77(6):523-525

4. Fujiyama S, Shibata J, Maeda S, Tanaka M, Noumaru S, Sato K et al (2003) Phase I clinical study of a novel lipophilic platinum complex (SM-11355) in patients with hepatocellular carcinoma refractory to cisplatin / lipiodol. Br J Cancer 89(9):1614-1619

5. Okusaka T, Okada S, Nakanishi T, Fujiyama S, Kubo Y (2004) Phase II trial of intra-arterial chemotherapy using a novel lipophilic platinum derivative (SM-11355) in patients with hepatocellular carcinoma. Invest New Drugs 22(2):169-176

6. Liver Cancer Study Group of Japan (1999) Survey and follow-up study of primary liver cancer in Japan. Report 13. Acta Hepatologica Japonica 40(5):288-300

7. Liver Cancer Study Group of Japan (2000) Survey and follow-up study of primary liver cancer in Japan. Report 14. Acta Hepatologica Japonica 41(12):799-811

8. Okusaka T, Okada S, Ishii H, Ikeda M, Nakasuka H, Nagahama H et al (1998) Transarterial chemotherapy with Zinostatin Stimalamer for hepatocellular carcinoma. Oncology 55(4):276-283

9. Okusaka T, Kasugai H, Shioyama Y, Tanaka K, Kudo M, Saisho H (2009) Transarterial chemotherapy alone versus transarterial chemoembolization for hepatocellular carcinoma: a randomized phase III trial. J Hepatol 51(6):1030-1036

10. Japanese Society of Liver Carcinoma (the Committee for Preparing Criteria for Evaluation of Multidisciplinary Treatment of Liver Carcinoma) (1994) Criteria for evaluation of direct effects of liver cancer treatment. Kanzo 35(2):193-205

11. Bruix J, Sherman M, Llovet JM, Beaugrand M, Lencioni R, Burroughs AK et al (2001) Clinical management of hepatocellular carcinoma. Conclusions of the Barcelona-2000 EASL conference. European Association for the Study of the Liver. J Hepatol 35 (3):421-430
12. Takayasu K, Arii S, Matsuo N, Yoshikawa M, Ryu M, Takasaki K et al (2000) Comparison of CT findings with resected specimens after chemoembolization with iodized oil for hepatocellular carcinoma. Am J Roentgenol 175(3):699-704

13. Okusaka T, Okada S, Ueno H, Ikeda M, Yoshimori M, Shimada K et al (2000) Evaluation of the therapeutic effect of transcatheter arterial embolization for hepatocellular carcinoma. Oncology 58 (4):293-299

14. Japan Society for Cancer Therapy (1986) The Japan Society for Cancer Therapy Criteria. J Jpn Soc Cancer Ther 21(5):929942

15. Japan Society for Cancer Therapy (1997) Toxicity grading criteria of the Japan Society for Cancer Therapy. J Jpn Soc Cancer Ther 1 (32):61-65

16. Takayasu K, Shima Y, Muramatsu Y, Moriyama N, Yamada T, Makuuchi M et al (1987) Hepatocellular carcinoma: treatment with intraarterial iodized oil with and without chemotherapeutic agents. Radiology 163(2):345-351

17. Hanada M, Baba A, Tsutsumishita Y, Noguchi T, Yamaoka T, Chiba $\mathrm{N}$ et al (2009) Intra-hepatic arterial administration with miriplatin suspended in an oily lymphographic agent inhitibs the growth of tumors implanted in rat livers by inducing platinum-DNA adducts to form and massive apoptosis. Cancer Chemother Pharmacol 64(3):473-483

18. Shibata J, Fujiyama S, Sato T, Kishimoto S, Fukushima S, Nakano M (1989) Hepatic arterial injection chemotherapy with cisplatin suspended in an oily lymphographic agent for hepatocellular carcinoma. Cancer 64(8):1586-1594

19. Sakaguchi T, Yoshimatsu S, Sagara K, Yamashita Y, Takahashi M (1998) Intra-arterial infusion of SMANCS for treatment of patients with hepatocellular carcinoma-adverse reactions and complications. Gan To Kagaku Ryoho 25(Suppl 1):64-69

20. Ikeda K, Saitoh S, Kobayashi M, Suzuki Y, Suzuki F, Tsubota A et al (2000) Hepatic vascular side effects of styrene maleic acid neocarzinostatin in the treatment of hepatocellular carcinoma. J Gastroenterol 35(5):353-360 